\title{
On Nehari disks and the inner radius
}

\author{
Leila Miller-Van Wieren
}

\begin{abstract}
Let $\mathrm{D}$ be a simply connected plane domain and $\mathrm{B}$ the unit disk. The inner radius of $\mathrm{D}, \sigma(\mathrm{D})$, is defined by $\sigma(\mathrm{D})=\sup \left\{a: a \geq 0,\left\|\mathrm{~S}_{f}\right\|_{\mathrm{D}} \leq a\right.$ implies $f$ is univalent in $\left.\mathrm{D}\right\}$. Here $\mathrm{S}_{f}$ is the Schwarzian derivative of $f, \rho_{\mathrm{D}}$ the hyperbolic density on $\mathrm{D}$ and $\left\|\mathrm{S}_{f}\right\|_{\mathrm{D}}=$ $\sup _{z \in \mathrm{D}}\left|\mathrm{S}_{f}(z)\right| \rho_{\mathrm{D}}^{-2}(z)$. Domains for which the value of $\sigma(\mathrm{D})$ is known include disks, angular sectors and regular polygons, as well as certain classes of rectangles and equiangular hexagons. All of the mentioned domains except non-convex angular sectors have an interesting property in common, namely that $\sigma(\mathrm{D})=2-\left\|\mathrm{S}_{h}\right\|_{\mathrm{B}}$, where $h$ maps $\mathrm{B}$ conformally onto $\mathrm{D}$. Because of the importance of this property for computing $\sigma(\mathrm{D})$, we say that $\mathrm{D}$ is a Nehari disk if $\sigma(\mathrm{D})=2-\left\|\mathrm{S}_{h}\right\|_{\mathrm{B}}$ holds.

This paper is devoted to the problem of characterizing Nehari disks. We give a necessary and sufficient condition for a domain to be a Nehari disk provided it is a regulated domain with convex corners.
\end{abstract}

Mathematics Subject Classification (2000). Primary 30C55; Secondary 30C20.

Keywords. Nehari disk, inner radius, univalence criteria.

\section{Introduction}

We use the symbol $\mathbb{C}$ to denote the complex plane and $\overline{\mathbb{C}}$ to denote the extended complex plane. Within $\overline{\mathbb{C}}$, we use the symbol $\mathrm{B}$ to refer to the unit disk $(\mathrm{B}=$ $\{z:|z|<1\})$ and $U$ for the upper half-plane $(U=\{z: \mathcal{I} \mathrm{m}(z)>0\})$. The symbol $\mathrm{D}$ will denote a domain in $\overline{\mathbb{C}}$ with at least two points on its boundary.

For $z \in \mathrm{B}$, the hyperbolic density of $\mathrm{B}$ at $z$ is the quantity $\rho_{\mathrm{B}}(z)$ given by $\rho_{\mathrm{B}}(z)=1 /\left(1-|z|^{2}\right)$. For a general simply connected domain $\mathrm{D}$, the hyperbolic density $\rho_{\mathrm{D}}$ is then defined in terms of $\rho_{\mathrm{B}}$ and $h: \mathrm{B} \longrightarrow \mathrm{D}$ where $h$ maps $\mathrm{B}$ conformally onto $\mathrm{D}$ (see [11, page 5$]$ ).

For $f$ holomorphic in $\mathrm{D} \subset \mathbb{C}$, with $f^{\prime}(z) \neq 0$ for $z \in \mathrm{D}$, the Schwarzian derivative $\mathrm{S}_{f}$, of $f$, is defined in $\mathrm{D}$ by $\mathrm{S}_{f}(z)=\left(f^{\prime \prime} / f^{\prime}\right)^{\prime}(z)-\frac{1}{2}\left(f^{\prime \prime} / f^{\prime}\right)^{2}(z)$. This definition can easily be extended to include locally univalent meromorphic functions. A detailed explanation of the extended definition can be found in [11, page 52]. To make our terminology more concise, locally univalent meromorphic 
functions will be refered to simply as locally univalent functions.

In order to discuss a univalence criterion for $f$ we introduce a norm for $\mathrm{S}_{f}$. Let $\mathrm{D}$ be a simply connected domain in $\overline{\mathbb{C}}$. For $f$ locally univalent in $\mathrm{D}$, we define the hyperbolic norm of $\mathrm{S}_{f}$ with respect to $\mathrm{D}$ by $\left\|\mathrm{S}_{f}\right\|_{\mathrm{D}}=\sup _{z \in \mathrm{D}}\left|\mathrm{S}_{f}(z)\right| \rho_{\mathrm{D}}^{-2}(z)$.

Now suppose $D$ is a simply connected domain in $\overline{\mathbb{C}}$. We define the inner radius of $\mathrm{D}, \sigma(\mathrm{D})$, by $\sigma(\mathrm{D})=\sup \left\{a: a \geq 0,\left\|\mathrm{~S}_{f}\right\|_{\mathrm{D}} \leq a\right.$ implies $f$ is univalent in $\mathrm{D}\}$.

All images of D under Möbius transformations have the same inner radius as D.

Nehari [14] and Hille [8] proved that $\sigma(\mathrm{B})=2$. Later, Lehtinen showed in [9] that $\sigma(\mathrm{D}) \leq 2$ for all simply connected domains in $\overline{\mathbb{C}}$ with equality occurring only when $\mathrm{D}$ is a disk in $\overline{\mathbb{C}}$ (i.e. an image of $\mathrm{B}$ under a Möbius transformation). The inner radius of a domain has another important meaning that is not apparent from its definition. Ahlfors and Weill [3] proved that if $f$ is locally univalent on $\mathrm{D}$ (a simply connected domain) with $\left\|\mathrm{S}_{f}\right\|_{\mathrm{D}}<\sigma(\mathrm{D})$, then $f$ is univalent and can be extended to a quasiconformal mapping of $\overline{\mathbb{C}}$. Ahlfors [1] and Gehring [5] proved that when $\mathrm{D}$ is a simply connected domain, $\sigma(\mathrm{D})>0$ if and only if $\mathrm{D}$ is a quasidisk.

Next, we list some known values of $\sigma(\mathrm{D})$. If $\mathrm{S}$ denotes the parallel strip defined as the image of $U$ under $h(z)=\log z$, then $\sigma(\mathrm{S})=0$. Lehto and Lehtinen have calculated the inner radii of angular sectors in [10] and [9]. If $\mathrm{A}_{k}=$ $\{z: z \in \mathbb{C}, 0<\arg z<k \pi\}$, then $\sigma\left(\mathrm{A}_{k}\right)=2 k^{2}$ for $0<k<1$ and $\sigma\left(\mathrm{A}_{k}\right)=4 k-$ $2 k^{2}$ for $1<k<2$. Another class of domains for which the inner radii have been calculated are regular polygons. Calvis [4], proved that $\sigma\left(\mathrm{P}_{n}\right)=2(n-2)^{2} / n^{2}$ where $\mathrm{P}_{n}$ is an open regular $n$-sided polygon.

In [12] we computed the inner radii for some classes of rectangles and equiangular hexagons. We proved that if $\mathrm{R}$ is a rectangle whose ratio of longer over shorter side is bounded from above by a specific constant $(\cong 1.52346 \ldots)$, then $\sigma(\mathrm{R})=1 / 2$, and if $H$ is an equiangular hexagon whose sides form the sequence baabaa with $b / a \leq 1.67117 \ldots$, then $\sigma(H)=8 / 9$. In the proofs of the just mentioned results the following simple but insightful lemma (see [13]) played a key role.

Lemma 1.1. If $\mathrm{D}$ is a simply connected domain and if $h$ maps $\mathrm{B}$ conformally onto $\mathrm{D}$, then $\sigma(\mathrm{D}) \geq 2-\left\|\mathrm{S}_{h}\right\|_{\mathrm{B}}$.

Proof. Suppose $f$ is locally univalent on $\mathrm{D}$ with $\left\|\mathrm{S}_{f}\right\|_{\mathrm{D}} \leq 2-\left\|\mathrm{S}_{h}\right\|_{\mathrm{B}}$. Then, $f \circ h$ is locally univalent on $\mathrm{B}$ and $\left\|\mathrm{S}_{f \circ h}\right\|_{\mathrm{B}}=\left\|\mathrm{S}_{f}-\mathrm{S}_{h^{-1}}\right\|_{\mathrm{D}} \leq\left\|\mathrm{S}_{f}\right\|_{\mathrm{D}}+\left\|\mathrm{S}_{h^{-1}}\right\|_{\mathrm{D}}=$ $\left\|\mathrm{S}_{f}\right\|_{\mathrm{D}}+\left\|\mathrm{S}_{h}\right\|_{\mathrm{B}} \leq 2$ (see [11]). This implies that $f \circ h$ is univalent on $\mathrm{B}$ and hence $f$ is univalent on $\mathrm{D}$. Thus $\sigma(\mathrm{D}) \geq 2-\left\|\mathrm{S}_{h}\right\|_{\mathrm{B}}$.

It turns out that the lower bound $2-\left\|\mathrm{S}_{h}\right\|_{\mathrm{B}}$ is equal to $\sigma(\mathrm{D})$ in the case of many domains for which $\sigma(\mathrm{D})$ is known-disks, parallel strips, convex angular sectors and regular polygons, as well as the mentioned classes of rectangles and equiangular hexagons (see [13] and [12]). Moreover, this yields a good method for computing $\sigma(\mathrm{D})$ for many domains. As will be seen later, computing $\sigma(\mathrm{D})$ for 
some domains can be based on merely understanding the behavior of the Riemann mapping $h$. This prompted us to introduce a special name for these domains (we first introduced it in [12]). A simply connected domain D in $\overline{\mathbb{C}}$ is called a Nehari disk if

$$
\sigma(\mathrm{D})=2-\left\|\mathrm{S}_{h}\right\|_{\mathrm{B}},
$$

where $h$ maps B conformally onto D. Nehari disks are essentially domains for which the application of the Nehari univalence criterion to $\left\|\mathrm{S}_{f \circ h}\right\|_{\mathrm{B}}=\left\|\mathrm{S}_{f}-\mathrm{S}_{h^{-1}}\right\|_{\mathrm{D}}$ gives the best possible result (and hence the name Nehari). Disks, parallel strips, convex angular sectors, regular polygons and the mentioned rectangles and equiangular hexagons are all Nehari disks. Of course, there exist many simply connected domains which are not Nehari disks. From the calculations in Lehto [10] and Lehtinen [9], one can easily see that the angular sectors $\mathrm{A}_{k}$ with $1<k<2$ are not Nehari disks. Also, from the presentation in Lehto [11, pages 60-61] we can conclude that there are many domains $\mathrm{D}$ for which $\left\|\mathrm{S}_{h}\right\|_{\mathrm{B}}>2$, where $h: \mathrm{B} \longrightarrow \mathrm{D}$ is the Riemann mapping. No such domain can be a Nehari disk.

In [13], it is demonstrated that some results previously known to hold for B are valid for all Nehari disks. We mention one of these generalizations here, as we will use it later on.

Theorem 1.2. Suppose D is a Nehari disk. If $f$ is a locally univalent function on $\mathrm{D}$ and if $\left\|\mathrm{S}_{f}\right\|_{\mathrm{D}} \leq \sigma(\mathrm{D})$, then $f(\mathrm{D})$ is a Jordan domain or the image of the parallel strip S under a Möbius transformation.

This is a generalization of the analogous theorem of Gehring and Pommerenke [7] for the unit disk. We recall another result from [7].

Theorem 1.3 (Gehring-Pommerenke). Suppose $f: \mathrm{B} \longrightarrow \overline{\mathbb{C}}$ is locally univalent on $\mathrm{B}$ and $\limsup _{|z| \rightarrow 1}\left|\mathrm{~S}_{f}(z)\right|\left(1-|z|^{2}\right)^{2}<2$. If $f(\mathrm{~B})$ is a Jordan domain, then $f(\mathrm{~B})$ is a quasidisk.

In this paper we establish a necessary and sufficient condition for a domain to be a Nehari disk, provided it belongs to a large and well known class of domains. Before introducing our result, we note a useful fact about how $\sigma(\mathrm{D})$ is affected by standard convergence of domains. In [12], the following relation between the inner radii of $\mathrm{D}_{n}$ and $\mathrm{D}$ when $\left\{\mathrm{D}_{n}\right\}$ converges to $\mathrm{D}$ in the sense of Carathéodory kernel convergence is demonstrated (for the definition of this convergence see $[16$, page 13]).

Theorem 1.4. If $\mathrm{D}_{n}$ and $\mathrm{D}$ are simply connected domains and if $\mathrm{D}_{n} \longrightarrow \mathrm{D}$ with respect to $w_{0}$, then $\sigma(\mathrm{D}) \geq \limsup _{n \rightarrow \infty} \sigma\left(\mathrm{D}_{n}\right)$.

\section{Main result}

In the introduction we saw that the definition of Nehari disks springs naturally from the problem of calculating $\sigma(\mathrm{D})$ for domains $\mathrm{D}$. That computation could be done using just the Riemann mapping, provided we knew that the domain 
was a Nehari disk. So, the question that comes to mind is: "Can Nehari disks be characterized in some interesting way?" Many domains in the applications of conformal mapping are bounded by finitely many smooth arcs that may form corners or may go to infinity. By restricting attention to a class of domains known as regulated domains, subject to the added constraint of having all convex corners, a class wide enough to include all the domains described earlier, we are able to obtain a necessary and sufficient condition for a domain to be a Nehari disk.

We begin with some definitions (see [16, pages 59-64]). A real-valued function $\beta$ on an interval $\mathrm{I}$ in $\mathbb{R}$ is said to be regulated if the one-sided limits $\beta(t-)=$ $\lim _{\tau \rightarrow t-} \beta(\tau)$ and $\beta(t+)=\lim _{\tau \rightarrow t+} \beta(\tau)$ exist for $t \in \mathrm{I}$ (in case $t$ is an endpoint of I only one of the above limits applies). Here and in what follows, we will assume that $\mathrm{D}$ is a Jordan domain and that $h$ maps $\mathrm{B}$ conformally onto $\mathrm{D}$. Then $h$ can be extended as a homeomorphism of $\overline{\mathrm{B}}$ onto $\overline{\mathrm{D}}$, for which we retain the notation $h$. We shall use the parametrization of $\partial \mathrm{D}$ given by $h\left(e^{i t}\right)$, for $0 \leq t \leq 2 \pi$ and periodically extended for $t \in \mathbb{R}$. We present three slightly modified definitions from [16], as we are concerned with Jordan domains only. We say that $\partial \mathrm{D}$ has a corner of opening $\alpha \pi,(0 \leq \alpha \leq 2)$ at $h\left(e^{i \xi}\right) \neq \infty,(\xi \in[0,2 \pi])$ if

$$
\arg \left[h\left(e^{i t}\right)-h\left(e^{i \xi}\right)\right] \longrightarrow \begin{cases}\beta & \text { as } t \rightarrow \xi+, \\ \beta+\alpha \pi & \text { as } t \rightarrow \xi-\end{cases}
$$

(It is assumed that $\arg z$ takes on values in $[0,2 \pi)$.) We also say that $\partial \mathrm{D}$ has a forward half-tangent of direction angle $\beta$ and a backward half-tangent of direction angle $\beta+\alpha \pi$ in this case. A corner and half-tangents at $\infty$ are defined by means of a preliminary inversion.

A Jordan domain $\mathrm{D}$ in $\mathbb{C}$ is said to be a regulated domain if

$$
\beta(t)= \begin{cases}\lim _{\tau \rightarrow t+} \arg \left[h\left(e^{i \tau}\right)-h\left(e^{i t}\right)\right] & \text { for } h\left(e^{i t}\right) \neq \infty \\ \lim _{\tau \rightarrow t+} \arg \left(h\left(e^{i \tau}\right)\right)+\pi & \text { for } h\left(e^{i t}\right)=\infty\end{cases}
$$

exists for all $t$ and defines a regulated function.

As discussed in [16], $\beta(t)$ is equal to $\beta(t+)$ and represents the direction angle of the forward half-tangent of $\partial \mathrm{D}$ at $h\left(e^{i t}\right)$; the limit $\beta(t-)$ represents the direction angle of the backward half-tangent at $h\left(e^{i t}\right)$. In addition, it is shown that $\beta(t+)=\beta(t-)$ for all but at most countably many $t$. We note the following useful proposition (see [16, page 60]).

Proposition 2.1. Suppose D is a regulated domain and $0<\epsilon<\pi$. If $|\beta(t)-\gamma|$ $<\epsilon$ and $h\left(e^{i t}\right) \neq \infty$ for $t \in \mathrm{I}$ where $\mathrm{I}$ is some interval in $\mathbb{R}$, then $\left|\arg \left[h\left(e^{i \tau}\right)-h\left(e^{i t}\right)\right]-\gamma\right|<\epsilon$ whenever $t, \tau \in \mathrm{I}$ and $t<\tau$.

As a regulated function on $[0,2 \pi], \beta$ can be written as $\beta=\beta_{\mathrm{c}}+\beta_{\mathrm{j}}$ where $\beta_{\mathrm{c}}$ is continuous on $[0,2 \pi]$ and $\beta_{\mathrm{j}}$ is constant on $[0,2 \pi]$ except for at most countably many jumps. The jumps clearly correspond to corners of $\partial \mathrm{D}$.

Regulated domains admit a simple representation formula for the conformal mapping $h, h(\mathrm{~B})=\mathrm{D}$. The following formula, due to Paatero [15], can also be 
found in [16]. If $h$ maps B conformally onto a regulated domain D, then

$$
\log h^{\prime}(z)=\log \left|h^{\prime}(0)\right|+\frac{i}{2 \pi} \int_{0}^{2 \pi} \frac{e^{i t}+z}{e^{i t}-z}\left(\beta(t)-t-\frac{\pi}{2}\right) d t
$$

for $z \in$ B. From (2.1), we obtain

$$
\begin{aligned}
\mathrm{S}_{h}(z)=\frac{2 i}{\pi} \int_{0}^{2 \pi} \frac{e^{i t}}{\left(e^{i t}-z\right)^{3}}\left(\beta(t)-t-\frac{\pi}{2}\right) d t & \\
& +\frac{1}{2 \pi^{2}}\left(\int_{0}^{2 \pi} \frac{e^{i t}}{\left(e^{i t}-z\right)^{2}}\left(\beta(t)-t-\frac{\pi}{2}\right) d t\right)^{2}
\end{aligned}
$$

for $z \in \mathrm{B}$, whenever $\mathrm{D}$ is regulated and $h, h: \mathrm{B} \longrightarrow \mathrm{D}$, is conformal.

Now we are ready to state our result.

Theorem 2.2. Suppose that D is a regulated domain with convex corners and that $h$ maps B conformally onto D. Then, D is a Nehari disk if and only if

$$
\limsup _{|z| \rightarrow 1}\left|\mathrm{~S}_{h}(z)\right|\left(1-|z|^{2}\right)^{2}=\left\|\mathrm{S}_{h}\right\|_{\mathrm{B}}
$$

Since the proofs of the two directions are different, we will address them in separate sections restating the above result as two separate theorems.

\section{The sufficient condition}

We begin by proving two lemmas that describe the behavior of the Schwarzian derivative of the mapping $h$ near $\partial \mathrm{B}$.

Lemma 3.1. Suppose that D is a regulated domain, that $h$ maps B conformally onto $\mathrm{D}$ and $\beta$ is as defined earlier. If $\zeta \in[0,2 \pi]$ and if $\beta$ is continuous at $\zeta$, then $\lim _{z \rightarrow e^{i \zeta}}\left|\mathrm{S}_{h}(z)\right|\left(1-|z|^{2}\right)^{2}=0$.

Proof. By applying a preliminary rotation we may assume that $\zeta \in(0,2 \pi)$. In view of (2.2), we begin by showing that

$$
\lim _{z \rightarrow e^{i \zeta}} \int_{0}^{2 \pi} \frac{e^{i t}\left(1-|z|^{2}\right)^{2}}{\left(e^{i t}-z\right)^{3}}\left(\beta(t)-t-\frac{\pi}{2}\right) d t=0
$$

Let $c=\beta(\zeta)$. First we will verify that $\lim _{z \rightarrow e^{i \zeta}} \int_{0}^{2 \pi} \frac{e^{i t}\left(1-|z|^{2}\right)^{2}}{\left(e^{i t}-z\right)^{3}}(\beta(t)-c) d t=0$.

Fix $\epsilon>0$. Since $\beta$ is continuous at $\zeta$, we can choose $\delta>0$ so that $\zeta-\delta<t<$ 
$\zeta+\delta \longrightarrow|\beta(t)-c|<\frac{\epsilon}{8 \pi}$. Next

$$
\begin{aligned}
\left|\int_{\zeta-\delta}^{\zeta+\delta} \frac{e^{i t}\left(1-|z|^{2}\right)^{2}}{\left(e^{i t}-z\right)^{3}}(\beta(t)-c) d t\right| & \leq \int_{\zeta-\delta}^{\zeta+\delta} \frac{\left(1-|z|^{2}\right)}{\left|e^{i t}-z\right|^{2}} \frac{\left(1-|z|^{2}\right)}{\left|e^{i t}-z\right|}|\beta(t)-c| d t \\
& \leq 2 \cdot \frac{\epsilon}{8 \pi} \int_{\zeta-\delta}^{\zeta+\delta} \frac{\left(1-|z|^{2}\right)}{\left|e^{i t}-z\right|^{2}} d t<\frac{\epsilon}{4 \pi} \cdot 2 \pi<\frac{\epsilon}{2}
\end{aligned}
$$

(using the Poisson representation formula [2, page 167]) for $z \in \mathrm{B}$. Let $\mathrm{A}_{\delta}=$ $[0, \zeta-\delta] \cup[\zeta+\delta, 2 \pi]$. Then, for $t \in \mathrm{A}_{\delta}$ and $z \in \mathrm{B},\left|z-e^{i \zeta}\right|<\left|e^{i\left(\zeta+\frac{\delta}{2}\right)}-e^{i \zeta}\right|:$

$$
\begin{aligned}
& \lim _{z \rightarrow e^{i \zeta}} \frac{e^{i t}\left(1-|z|^{2}\right)^{2}}{\left(e^{i t}-z\right)^{3}}(\beta(t)-c)=0 \text { and } \\
& \qquad\left|\frac{e^{i t}\left(1-|z|^{2}\right)^{2}}{\left(e^{i t}-z\right)^{3}}(\beta(t)-c)\right| \text { is uniformly bounded. }
\end{aligned}
$$

From the Dominated Convergence Theorem (see [17]),

$\lim _{z \rightarrow e^{i \zeta}} \int_{\mathrm{A}_{\delta}} \frac{e^{i t}\left(1-|z|^{2}\right)^{2}}{\left(e^{i t}-z\right)^{3}}(\beta(t)-c) d t=0$. Hence, we can fix $\delta^{\prime}>0$ so that for $z \in \mathrm{B}$ and $\left|z-e^{i \zeta}\right|<\delta^{\prime}$,

$$
\left|\int_{\mathrm{A}_{\delta}} \frac{e^{i t}\left(1-|z|^{2}\right)^{2}}{\left(e^{i t}-z\right)^{3}}(\beta(t)-c) d t\right|<\frac{\epsilon}{2} .
$$

Now, from (3.1), (3.2) and the triangle inequality,

$\left|\int_{0}^{2 \pi} \frac{e^{i t}\left(1-|z|^{2}\right)^{2}}{\left(e^{i t}-z\right)^{3}}(\beta(t)-c) d t\right|<\epsilon$ for $z \in \mathrm{B},\left|z-e^{i \zeta}\right|<\delta^{\prime}$. Since $\epsilon>0$ was arbitrary, we have shown that

$$
\lim _{z \rightarrow e^{i \zeta}} \int_{0}^{2 \pi} \frac{e^{i t}\left(1-|z|^{2}\right)^{2}}{\left(e^{i t}-z\right)^{3}}(\beta(t)-c) d t=0
$$


Now using integration by parts we get

$$
\begin{aligned}
\left|\int_{0}^{2 \pi} \frac{e^{i t}\left(1-|z|^{2}\right)^{2}}{\left(e^{i t}-z\right)^{3}}\left(c-t-\frac{\pi}{2}\right) d t\right| & =\left|-i \pi \frac{\left(1-|z|^{2}\right)^{2}}{(1-z)^{2}}+i \int_{0}^{2 \pi} \frac{\left(1-|z|^{2}\right)^{2}}{2\left(e^{i t}-z\right)^{2}} d t\right| \\
& \leq \pi \frac{\left(1-|z|^{2}\right)^{2}}{|1-z|^{2}}+\int_{0}^{2 \pi} \frac{\left(1-|z|^{2}\right)^{2}}{2\left|e^{i t}-z\right|^{2}} d t
\end{aligned}
$$

for $z \in \mathrm{B}$. Again, from the Poisson representation formula, it is clear that

$$
\lim _{z \rightarrow e^{i \zeta}} \int_{0}^{2 \pi} \frac{e^{i t}\left(1-|z|^{2}\right)^{2}}{\left(e^{i t}-z\right)^{3}}\left(c-t-\frac{\pi}{2}\right) d t=0
$$

Thus from (3.3) and (3.4), $\lim _{z \rightarrow e^{i \zeta}} \int_{0}^{2 \pi} \frac{e^{i t}\left(1-|z|^{2}\right)^{2}}{\left(e^{i t}-z\right)^{3}}\left(\beta(t)-t-\frac{\pi}{2}\right) d t=0$. In a completely analogous fashion, it can be verified that

$\lim _{z \rightarrow e^{i \zeta}} \int_{0}^{2 \pi} \frac{e^{i t}\left(1-|z|^{2}\right)}{\left(e^{i t}-z\right)^{2}}\left(\beta(t)-t-\frac{\pi}{2}\right) d t=0$. Thus from (2.2),

$\lim _{z \rightarrow e^{i \zeta}}\left|\mathrm{S}_{h}(z)\right|\left(1-|z|^{2}\right)^{2}=0$.

Next, we show a general lemma describing the behavior of the Schwarzian derivative of the mapping $h$ near $\partial \mathrm{B}$.

Lemma 3.2. Suppose $h$ maps B conformally onto a regulated domain D where $\beta=\beta_{c}+\beta_{j}$ (as defined earlier) and $\beta_{j}$ has jumps $\sigma_{k} \pi$ at $t_{k} \in[0,2 \pi]$ for $k=1,2 \ldots$ (there are at most countably many jumps). Then

$$
\limsup _{|z| \rightarrow 1}\left|S_{h}(z)\right|\left(1-|z|^{2}\right)^{2}=\sup _{k}\left|4 \sigma_{k}-2 \sigma_{k}^{2}\right|=\sup _{k}\left|2-2 \alpha_{k}^{2}\right|
$$

where $\alpha_{k}=1-\sigma_{k}$ for all $k$.

Proof. Suppose $k$ is arbitrarily fixed. We will calculate $\limsup _{z \rightarrow e^{i t_{k}}}\left|\mathrm{~S}_{h}(z)\right|\left(1-|z|^{2}\right)^{2}$.

By applying a preliminary rotation we may assume that $t_{k} \in(0,2 \pi)$. Let $\beta_{k}(t)=\beta(t)$ for $t \in\left[0, t_{k}\right), \beta_{k}(t)=\beta(t)-\sigma_{k} \pi$ for $t \in\left[t_{k}, 2 \pi\right)$. Since $\beta$ is regulated on $[0,2 \pi]$ and $\beta\left(t_{k}\right)=\beta\left(t_{k}+\right)$, we deduce that $\beta_{k}$ is regulated on 
$[0,2 \pi]$ and is continuous at $t_{k}$. Let $\beta_{\sigma_{k}}=\beta-\beta_{k}$ on $[0,2 \pi]$. If

$$
\begin{aligned}
\mathrm{I}(z) & =\int_{0}^{2 \pi} \frac{e^{i t}}{\left(e^{i t}-z\right)^{3}}\left(\beta_{k}(t)-t-\frac{\pi}{2}\right) d t+\int_{0}^{2 \pi} \frac{e^{i t}}{\left(e^{i t}-z\right)^{3}} \beta_{\sigma_{k}}(t) d t \\
& =\int_{0}^{2 \pi} \frac{e^{i t}}{\left(e^{i t}-z\right)^{3}}\left(\beta_{k}(t)-t-\frac{\pi}{2}\right) d t+\frac{\sigma_{k} \pi i}{2} \frac{\left(e^{i t_{k}}-1\right)\left(e^{i t_{k}}+1-2 z\right)}{(1-z)^{2}\left(e^{i t_{k}}-z\right)^{2}}
\end{aligned}
$$

and $\quad \mathrm{J}(z)=\int_{0}^{2 \pi} \frac{e^{i t}}{\left(e^{i t}-z\right)^{2}}\left(\beta_{k}(t)-t-\frac{\pi}{2}\right) d t+\int_{0}^{2 \pi} \frac{e^{i t}}{\left(e^{i t}-z\right)^{2}} \beta_{\sigma_{k}}(t) d t$

$$
=\int_{0}^{2 \pi} \frac{e^{i t}}{\left(e^{i t}-z\right)^{2}}\left(\beta_{k}(t)-t-\frac{\pi}{2}\right) d t+\sigma_{k} \pi i \frac{e^{i t_{k}}-1}{(1-z)\left(e^{i t_{k}}-z\right)},
$$

then from (2.2) we obtain $\mathrm{S}_{h}(z)\left(1-|z|^{2}\right)^{2}=\left[\frac{2 i}{\pi} \mathrm{I}(z)+\frac{1}{2 \pi^{2}}(\mathrm{~J}(z))^{2}\right]\left(1-|z|^{2}\right)^{2}$ for $z \in \mathrm{B}$. Thus

$$
\begin{aligned}
\mathrm{S}_{h}(z)\left(1-|z|^{2}\right)^{2} \\
=\quad\left[\frac{2 i}{\pi} \int_{0}^{2 \pi} \frac{e^{i t}}{\left(e^{i t}-z\right)^{3}}\left(\beta_{k}(t)-t-\frac{\pi}{2}\right) d t\right. \\
\left.\quad+\frac{1}{2 \pi^{2}}\left(\int_{0}^{2 \pi} \frac{e^{i t}}{\left(e^{i t}-z\right)^{2}}\left(\beta_{k}(t)-t-\frac{\pi}{2}\right) d t\right)^{2}\right]\left(1-|z|^{2}\right)^{2} \\
+\left[\sigma_{k} \frac{e^{i t_{k}}+1-2 z}{1-z}-\frac{1}{2} \sigma_{k}^{2} \frac{1-e^{i t_{k}}}{1-z}\right]\left[\frac{1-e^{i t_{k}}}{1-z}\right]\left[\frac{1-|z|^{2}}{e^{i t_{k}}-z}\right]^{2} \\
+\frac{\sigma_{k} i}{\pi}\left[\int_{0}^{2 \pi} \frac{e^{i t}\left(1-|z|^{2}\right)}{\left(e^{i t}-z\right)^{2}}\left(\beta_{k}(t)-t-\frac{\pi}{2}\right) d t\right]\left[\frac{e^{i t_{k}}-1}{(1-z)\left(e^{i t_{k}}-z\right)}\right]\left(1-|z|^{2}\right) .
\end{aligned}
$$

From the proof of Lemma 3.1, it is apparent that

$$
\begin{aligned}
\lim _{z \rightarrow e^{i t} k}\left[\frac{2 i}{\pi} \int_{0}^{2 \pi}\right. & \frac{e^{i t}}{\left(e^{i t}-z\right)^{3}}\left(\beta_{k}(t)-t-\frac{\pi}{2}\right) d t \\
& \left.+\frac{1}{2 \pi^{2}}\left(\int_{0}^{2 \pi} \frac{e^{i t}}{\left(e^{i t}-z\right)^{2}}\left(\beta_{k}(t)-t-\frac{\pi}{2}\right) d t\right)^{2}\right]\left(1-|z|^{2}\right)^{2}=0
\end{aligned}
$$

and that

$$
\begin{aligned}
\lim _{z \rightarrow e^{i t_{k}}} \frac{\sigma_{k} i}{\pi}\left[\int _ { 0 } ^ { 2 \pi } \frac { e ^ { i t } ( 1 - | z | ^ { 2 } ) } { ( e ^ { i t } - z ) ^ { 2 } } \left(\beta_{k}(t)-t-\right.\right. & \left.\left.\frac{\pi}{2}\right) d t\right] \\
& \cdot\left[\frac{e^{i t_{k}}-1}{(1-z)\left(e^{i t_{k}}-z\right)}\right]\left(1-|z|^{2}\right)=0 .
\end{aligned}
$$


From (3.5) and the above equations we get

$$
\begin{aligned}
& \limsup _{z \rightarrow e^{i t_{k}}}\left|\mathrm{~S}_{h}(z)\right|\left(1-|z|^{2}\right)^{2} \\
& =\limsup _{z \rightarrow e^{i t_{k}}}\left[\sigma_{k} \frac{e^{i t_{k}}+1-2 z}{1-z}-\frac{1}{2} \sigma_{k}^{2} \frac{1-e^{i t_{k}}}{1-z}\right]\left[\frac{1-e^{i t_{k}}}{1-z}\right]\left[\frac{1-|z|^{2}}{e^{i t_{k}}-z}\right]^{2} \\
& =\left|4 \sigma_{k}-2 \sigma_{k}^{2}\right| .
\end{aligned}
$$

Since $k$ was arbitrary, from Lemma 3.1 we deduce that

$$
\limsup _{|z| \rightarrow 1}\left|S_{h}(z)\right|\left(1-|z|^{2}\right)^{2}=\sup _{k}\left|4 \sigma_{k}-2 \sigma_{k}^{2}\right|=\sup _{k}\left|2-2 \alpha_{k}^{2}\right|
$$

where $\alpha_{k}=1-\sigma_{k}$.

In view of Lemma 3.2, if we know that $\limsup _{|z| \rightarrow 1}\left|\mathrm{~S}_{h}(z)\right|\left(1-|z|^{2}\right)^{2}=\left\|\mathrm{S}_{h}\right\|_{\mathrm{B}}$, then $\left\|\mathrm{S}_{h}\right\|_{\mathrm{B}}$ can be explicitly calculated for regulated D. Consequently, we would know the value of $2-\left\|\mathrm{S}_{h}\right\|_{\mathrm{B}}-\mathrm{a}$ lower bound for $\sigma(\mathrm{D})$. This becomes significant when combined with the next lemma which shows that the inner radius of a domain with a corner on the boundary is bounded from above by the inner radius of the angular sector corresponding to it.

Lemma 3.3. Suppose that D is a Jordan domain, that $h$ maps B onto D conformally and that $\partial \mathrm{D}$ has a corner of opening $\alpha \pi$ for some $\alpha, 0<\alpha<2$. Then

$$
\sigma(\mathrm{D}) \leq \begin{cases}2 \alpha^{2} & \text { if } 0<\alpha \leq 1 \\ 4 \alpha-2 \alpha^{2} & \text { if } 1 \leq \alpha<2\end{cases}
$$

Proof. Since $h$ is sense-preserving, D remains on the left side of $\partial \mathrm{D}$ as this curve is traced out by $h\left(e^{i t}\right)$ as $t$ increases. Let $\xi \in[0,2 \pi]$ be the point for which $\partial \mathrm{D}$ has a corner of opening $\alpha \pi$ at $h\left(e^{i \xi}\right)$. By applying a suitable Möbius transformation we may assume without loss of generality that $h\left(e^{i \xi}\right)=0$ and that the backward and forward half-tangents at $h\left(e^{i \xi}\right)$ coincide with $p=\left\{z=r e^{i \alpha \pi}: r>0\right\}$ and $q=\{z=r: r>0\}$, respectively. In other words, the corner formed by the two half-tangents at $h\left(e^{i \xi}\right)$ is $\mathrm{A}_{\alpha}$. Now for $X \subseteq \mathbb{C}, c \in \mathbb{R}$ we define $c X=\{c z: z \in X\}$.

We will show that $n \mathrm{D} \longrightarrow \mathrm{A}_{\alpha}$ with respect to $w_{0}$, for some $w_{0} \in \mathrm{A}_{\alpha}$. The proof is based on the following well-known fact.

If $0<\epsilon<\alpha \pi / 2$, then there exists $r_{\epsilon}>0$ such that the triangular sector

$$
\mathrm{T}_{\epsilon}=\left\{z=r e^{i \theta}: \epsilon<\theta<\alpha \pi-\epsilon, 0<r<r_{\epsilon}\right\}
$$

is contained in D.

We can choose $w_{0} \in \mathrm{T}_{\frac{\alpha \pi}{4}}$. Then $w_{0} \in \mathrm{T}_{\frac{\alpha \pi}{4}} \subseteq n \mathrm{~T} \frac{\alpha \pi}{4} \subseteq n \mathrm{D}$ for $n \in \mathbb{N}$, so there is a neighborhood of $w_{0}$ contained in $n \mathrm{D}$ for $n \in \mathbb{N}$. 
Given any $w \in \mathrm{A}_{\alpha}$, we can choose $\epsilon, 0<\epsilon<\alpha \pi / 4$ and $n_{0}, n_{0} \in \mathbb{N}$ such that $w / n_{0} \in \mathrm{T}_{\epsilon}$. Then $w \in n_{0} \mathrm{~T}_{\epsilon} \subseteq n \mathrm{~T}_{\epsilon} \subseteq n \mathrm{D}$ for $n \in \mathbb{N}, n \geq n_{0}$.

Finally, we check the boundary condition of kernel convergence. Suppose $w \in$ $\partial \mathrm{A}_{\alpha}$ is arbitrarily fixed. We need to show that $\operatorname{dist}(w, \partial(n \mathrm{D})) \longrightarrow 0$. Suppose this was not true. Without loss of generality we can assume that $w \in p$. For $\epsilon>0$ let

$$
\mathrm{S}_{\epsilon}=\{z:|| z|-| w||<\epsilon,|\arg z-\alpha \pi|<\epsilon\} .
$$

By our assumption, there exists $\epsilon>0$ and an increasing sequence $\left\{n_{i}\right\}, n_{i} \in \mathbb{N}$ for $i \in \mathbb{N}$ such that $\mathrm{S}_{\epsilon} \bigcap \partial\left(n_{i} \mathrm{D}\right)=\emptyset$ for $i \in \mathbb{N}$. Consequently $\frac{1}{n_{i}} \mathrm{~S}_{\epsilon} \bigcap \partial \mathrm{D}=\emptyset$ for $i \in \mathbb{N}$ - this contradicts the fact that $p$ is the backward half-tangent of $\partial \mathrm{D}$ at $h\left(e^{i \xi}\right)$. Thus dist $(w, \partial(n \mathrm{D})) \longrightarrow 0$ for $w \in \partial \mathrm{A}_{\alpha}$.

This completes the proof that $n \mathrm{D} \longrightarrow \mathrm{A}_{\alpha}$ with respect to $w_{0}$. Since $\sigma(n \mathrm{D})=$ $\sigma(\mathrm{D})$ for $n \in \mathbb{N}$, if we consider the known values of $\sigma\left(\mathrm{A}_{\alpha}\right)$ and Theorem 1.4, the statement of the lemma follows.

We are now prepared to present the sufficiency portion of the main result.

Theorem 3.4. Suppose that D is a regulated domain with convex corners and that $h$ maps B conformally onto D. If $\limsup _{|z| \longrightarrow 1}\left|\mathrm{~S}_{h}(z)\right|\left(1-|z|^{2}\right)^{2}=\left\|\mathrm{S}_{h}\right\|_{\mathrm{B}}$, then $\mathrm{D}$ is a Nehari disk.

Proof. Suppose $\beta=\beta_{c}+\beta_{j}$ and $\sigma_{k} \pi, k=1,2 \ldots$ are as in Lemma 3.2. Each jump $\sigma_{k} \pi$ corresponds to a corner $\alpha_{k} \pi$, where $\alpha_{k}=1-\sigma_{k}$ if the corner is at a finite point on $\partial \mathrm{D}$ and $\alpha_{k}=\sigma_{k}-1$ if the corner is at infinity. Also $0<\alpha_{k}<1$ for each $k$. Lemma 3.3 implies that $\sigma(\mathrm{D}) \leq 2 \alpha_{k}^{2}$ for each $k$ and hence $\sigma(\mathrm{D}) \leq \inf _{k} 2 \alpha_{k}^{2}$. On the other hand, Lemma 3.2 and the assumption of the theorem imply that $\left\|\mathrm{S}_{h}\right\|_{\mathrm{B}}=\sup \left(2-2 \alpha_{k}^{2}\right)$. From the above and Lemma 1.1, we get $\sigma(\mathrm{D}) \geq 2-\sup _{k}\left(2-2 \alpha_{k}^{2}\right) \geq \inf _{k} 2 \alpha_{k}^{2}$. Hence, it follows that $\sigma(\mathrm{D})=\inf _{k} 2 \alpha_{k}^{2}$ and that $\mathrm{D}$ is a Nehari disk.

In the proof of the above theorem we have also verified the following corollary.

Corollary 3.5. Suppose D satisfies the assumptions of Theorem 3.4. If $\partial \mathrm{D}$ has corners $\alpha_{k} \pi, k=1,2 \ldots$, then $\sigma(\mathrm{D})=\inf _{k} 2 \alpha_{k}^{2}$.

\section{The necessary condition}

Before moving on to the necessary condition, we establish some preliminary facts.

Lemma 4.1. Suppose D is a regulated quasidisk and $\left\{\lambda_{n}\right\}$ is a sequence of similarity transformations such that there are points $z_{1}, z_{2}$ outside of all $\lambda_{n}(\mathrm{D})$ and $\lambda_{n}(\mathrm{D}) \longrightarrow \mathrm{D}_{0}$ with respect to some $z_{0} \in \mathrm{D}_{0}$, where $\mathrm{D}_{0}$ is some domain.

Then, $\mathrm{D}_{0}$ is Möbius equivalent to $\mathrm{D}$, or $\mathrm{D}_{0}$ is a half-plane, or $\mathrm{D}_{0}$ is Möbius equivalent to $\mathrm{A}_{\alpha}$, where $\alpha \pi$ is a corner of $\partial \mathrm{D}$. 
Proof. Let $h: \mathrm{B} \longrightarrow \mathrm{D}$ denote a fixed conformal mapping with $h(\mathrm{~B})=\mathrm{D}$. During the course of the proof, we will replace $\left\{\lambda_{n}\right\}$ by a subsequence and relabel it several times - a process that does not affect the assumptions of the lemma. Since $\left\{\lambda_{n}\right\}$ is a normal family in D, after passing to a subsequence and relabeling, we can assume that either $\lambda_{n} \longrightarrow \lambda$ locally uniformly in $\overline{\mathbb{C}}$, where $\lambda$ is a similarity transformation, or $\lambda_{n} \longrightarrow c$ locally uniformly in $\mathrm{D}$, where $c$ is a constant in $\overline{\mathbb{C}}$.

In the first case, it is easy to verify that $D_{0}=\lambda(D)$ so $D_{0}$ is Möbius equivalent to $\mathrm{D}$, proving the lemma.

Suppose $\lambda_{n} \longrightarrow c$ locally uniformly in D. For $n \in \mathbb{N}, \lambda_{n}(z)=a_{n} z+b_{n}$ for some complex $a_{n}, b_{n} ; a_{n} \neq 0$. By passing to a subsequence and relabeling, we may assume that $\arg a_{n} \longrightarrow \theta \pi$ for some $\theta \in[0,2]$.

After passing to a further subsequence, we may assume that $h^{-1} \circ \lambda_{n}^{-1} \longrightarrow w_{0}$ locally uniformly in $\mathrm{D}_{0}$, for some $w_{0} \in \partial \mathrm{B}$. Since $\mathrm{D}$ is a quasidisk, we can assume that $h$ is a homeomorphism of $\overline{\mathrm{B}}$ onto $\overline{\mathrm{D}}$.

Now, for $n \in \mathbb{N}$, we can find a conformal mapping $g_{n}: \mathrm{B} \longrightarrow \lambda_{n}(\mathrm{D})$ (continuously extended to the boundary) with $g_{n}(\mathrm{~B})=\lambda_{n}(\mathrm{D})$, such that:

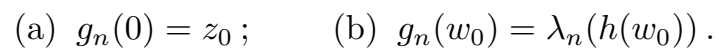

Modulo extraction of another subsequence, we may assume that $g_{n} \longrightarrow g$ locally uniformly in B, where $g$ is conformal and $g(\mathrm{~B})=\mathrm{D}_{0}$. Also, since all $\lambda_{n}(\mathrm{D})$ are $\mathrm{K}$-quasidisks for some fixed $\mathrm{K}$, we may extend each $g_{n}$ by reflection to a $\mathrm{K}^{2}$-quasiconformal mapping of $\overline{\mathbb{C}}$, and retaining the name $g_{n}$ for the extension, assume that $g_{n} \longrightarrow g$ locally uniformly in $\overline{\mathbb{C}}$, where $g$ is a $\mathrm{K}^{2}$-quasiconformal extension of the original mapping $g$.

Let $\mu_{n}=h^{-1} \circ \lambda_{n}^{-1} \circ g_{n}$. In B, $\mu_{n}$ is the restriction of a Möbius transformation, and (from (a) and (b)) $\lim _{n \rightarrow \infty} \mu_{n}(0)=w_{0}, \mu_{n}\left(w_{0}\right)=w_{0}, \mu_{n}(\mathrm{~B})=\mathrm{B}$. Consequently, after additional "pruning" and relabeling, we may assume that $\mu_{n}(z) \longrightarrow w_{0}$ for all but possibly one $z \in \overline{\mathrm{B}}$. Let $t_{0} \in[0,2 \pi)$ be the point for which $e^{i t_{0}}=w_{0}$. Without loss of generality, we may assume that $t_{0} \neq 0$. We have $\mu_{n}(z) \longrightarrow e^{i t_{0}}$ for all but possibly one $z \in \partial \mathrm{B}$ and $\mu_{n}\left(e^{i t_{0}}\right)=e^{i t_{0}}$ for each $n$.

Let $\xi_{0}=\sup \left\{\xi: \xi \in\left[t_{0}, t_{0}+2 \pi\right)\right.$ such that $\arg \left(\mu_{n_{k}}\left(e^{i \xi}\right)\right) \rightarrow t_{0}+$, for some $\left.\left\{n_{k}\right\}\right\}$. From the definition of $\xi_{0}$ and the fact that $\mu_{n}$ are all sense-preserving we conclude that:

(i)For any $t_{0}<t<\tau<\xi_{0}$, there exists a sequence $\left\{n_{k}\right\}$ such that $t_{0} \leq \arg \left(\mu_{n_{k}}\left(e^{i t}\right)\right) \leq \arg \left(\mu_{n_{k}}\left(e^{i \tau}\right)\right)$ and $\lim _{k \rightarrow \infty} \arg \left(\mu_{n_{k}}\left(e^{i \tau}\right)\right)=t_{0}+$;

(ii)For $\xi_{0}<t<t_{0}+2 \pi, \lim _{n \rightarrow \infty} \arg \left(\mu_{n}\left(e^{i t}\right)\right)=t_{0}-$.

( In case $\xi_{0}=t_{0}$ or $\xi_{0}=t_{0}+2 \pi$ only (ii) or (i) applies.)

Since D is regulated, from Proposition 2.1 we know that

$$
\begin{aligned}
\lim _{0<t<\tau<t_{0}, t \rightarrow t_{0}-} \arg \left[h\left(e^{i \tau}\right)-h\left(e^{i t}\right)\right] & =\beta\left(t_{0}-\right)=\gamma \pi \\
\lim _{t_{0}<t<\tau<2 \pi, \tau \rightarrow t_{0}+} & \arg \left[h\left(e^{i \tau}\right)-h\left(e^{i t}\right)\right]=\beta\left(t_{0}+\right)=\beta \pi
\end{aligned}
$$


for some $\gamma, \beta$ in $[0,2)$. Thus, for $t_{0}<t<\tau<\xi_{0}$, there exists $\left\{n_{k}\right\}$ such that

$$
\begin{aligned}
\arg \left[g\left(e^{i \tau}\right)-g\left(e^{i t}\right)\right]=\lim _{k \rightarrow \infty} \arg & {\left[g_{n_{k}}\left(e^{i \tau}\right)-g_{n_{k}}\left(e^{i t}\right)\right] } \\
& =\lim _{k \rightarrow \infty} \arg \left[a_{n_{k}}\left(h\left(\mu_{n_{k}}\left(e^{i \tau}\right)\right)-h\left(\mu_{n_{k}}\left(e^{i t}\right)\right)\right)\right]
\end{aligned}
$$

and consequently, since $\arg a_{n_{k}} \longrightarrow \theta \pi, \arg \left[g\left(e^{i \tau}\right)-g\left(e^{i t}\right)\right]=\beta \pi+\theta \pi$ (or $\beta \pi+\theta \pi-2 \pi)$. Analogously, for $\xi_{0}<t<\tau<t_{0}+2 \pi, \arg \left[g\left(e^{i \tau}\right)-g\left(e^{i t}\right)\right]=$ $\gamma \pi+\theta \pi \quad$ (or $\gamma \pi+\theta \pi-2 \pi)$. Since $\partial \mathrm{D}_{0}=\partial g(\mathrm{~B})$ is a Jordan curve, we conclude that $\mathrm{D}_{0}$ is a half-plane when $\gamma=\beta$, while $\mathrm{D}_{0}$ is the image of $\mathrm{A}_{\alpha}$ under a similarity transformation when $\gamma \neq \beta$, where $\alpha=1-(\beta-\gamma)$. As $\partial \mathrm{D}$ has a corner of opening $\alpha \pi$ in the second case, the proof is complete.

In the lemma that follows, we will demonstrate that (roughly speaking) a sequence of $\mathrm{K}_{n}$-quasidisks with $\mathrm{K}_{n} \longrightarrow \infty$ can, after passage to a subsequence, be "arranged" so that it converges to a non-Jordan domain. We will express this fact in terms of the three-point property (see [6]). First we introduce some notation. For $x, y \in \mathbb{C}$, let $(x, y)([x, y])$ denote the open (closed) line segment with endpoints $x$ and $y$ (note that $(x, y)=(y, x)$ in this notation). For a quasidisk D in $\overline{\mathbb{C}}$, set $d(\mathrm{D})=\inf \left\{\frac{|x-y|}{|x-z|}: x, y, z\right.$ satisfy $\left.(\star)\right\}$, where:

$(\star)-x, y, z$ are distinct points on $\partial \mathrm{D} \backslash\{\infty\}$ and $z$ is in the component of $\partial \mathrm{D} \backslash\{x, y\}$ with minimal diameter.

Lemma 4.2. Suppose $\left\{\mathrm{D}_{n}\right\}$ is a sequence of quasidisks such that $d\left(\mathrm{D}_{n}\right) \longrightarrow$ 0 . There exists a sequence of natural numbers $\left\{n_{k}\right\}$, a sequence of Möbius transformations $\left\{\mu_{n_{k}}\right\}$ and a domain $N$ containing $(0,1)$, such that:

(i) $N \subseteq \mu_{n_{k}}\left(\mathrm{D}_{n_{k}}\right)$ and $0,1, \infty \in \partial \mu_{n_{k}}\left(\mathrm{D}_{n_{k}}\right)$ for all $k$;

(ii) $\mu_{n_{k}}\left(\mathrm{D}_{n_{k}}\right) \longrightarrow \mathrm{D}^{\prime}$ (with respect to any point in $N$ ), where $\mathrm{D}^{\prime}$ is a nonJordan domain containing $N$.

Proof. Without loss of generality, we may assume that $\infty \in \partial \mathrm{D}_{n}$ for $n \in \mathbb{N}$. Let $d_{n}=d\left(\mathrm{D}_{n}\right)$ for $n \in \mathbb{N}$. Also, we may assume that $d_{n}<1$ for $n \in \mathbb{N}$. Now for $n \in \mathbb{N}$ we pick $x_{n}, y_{n}, z_{n}$ and $\epsilon_{n}>0$ so that:

(i) $x_{n}, y_{n}, z_{n}$ satisfy $(\star)$ applied to $\mathrm{D}_{n}$ and $z_{n}$ is such that $\left|x_{n}-z_{n}\right|$ is maximal;

(ii) $d_{n} \leq\left|x_{n}-y_{n}\right| /\left|x_{n}-z_{n}\right| \leq d_{n}\left(1+\epsilon_{n}\right)$;

(iii) $\epsilon_{n} \longrightarrow 0$.

Without loss of generality we can assume that, for each $n$, either $\left(x_{n}, y_{n}\right) \subseteq \mathrm{D}_{n}$ or $\left(x_{n}, y_{n}\right) \subseteq \overline{\mathbb{C}} \backslash \mathrm{D}_{n}$. (If not, replace $x_{n}, y_{n}$ with $x_{n}^{\prime}, y_{n}^{\prime}$, where $x_{n}^{\prime}, y_{n}^{\prime}$ are picked from the intersections of the components of $\partial \mathrm{D}_{n} \backslash\left\{z_{n}, \infty\right\}$, that contain $x_{n}, y_{n}$ respectively, with $\overline{\mathrm{B}\left(\frac{\left(x_{n}+y_{n}\right)}{2}, \frac{\left|x_{n}-y_{n}\right|}{2}\right)}$, so that $\left|x_{n}^{\prime}-y_{n}^{\prime}\right|$ is minimal, then replace $z_{n}$ with $z_{n}^{\prime}$ so that (i) holds for $x_{n}^{\prime}, y_{n}^{\prime}, z_{n}^{\prime}$. It is easy to find $\epsilon_{n}>0$ so that (ii) and (iii) hold.)

By passing to a subsequence and relabeling, we can assume that either $\left(x_{n}, y_{n}\right)$ $\subseteq \mathrm{D}_{n}$ for all $n$, or $\left(x_{n}, y_{n}\right) \subseteq \overline{\mathbb{C}} \backslash \mathrm{D}_{n}$ for all $n$. We give the proof for the first case only, as a modification of the same argument works in the second case. 
So assume that $\left(x_{n}, y_{n}\right) \subseteq \mathrm{D}_{n}$ for all $n$. For each $n$, choose a similarity transformation $\mu_{n}$ such that $\mu_{n}\left(\left[x_{n}, y_{n}\right]\right)=[0,1]$, and let $z_{n}^{\prime}=\mu_{n}\left(z_{n}\right)$. From (ii) it follows that:

$$
d_{n} \leq \frac{1}{\left|z_{n}^{\prime}\right|}=\frac{|0-1|}{\left|0-z_{n}^{\prime}\right|} \leq d_{n}\left(1+\epsilon_{n}\right)
$$

and that $z_{n}^{\prime}$ is in the bounded component of $\partial \mu_{n}\left(\mathrm{D}_{n}\right) \backslash\{0,1\}$. It is easy to verify that for each $z \in(0,1)$, we can fix $\delta_{z}>0$ so that $\mathrm{B}\left(z, \delta_{z}\right) \subseteq \mu_{n}\left(\mathrm{D}_{n}\right)$ for all $n$. We introduce some notation. Let $N=\bigcup_{z \in(0,1)} \mathrm{B}\left(z, \delta_{z}\right), \mathrm{V}=\bigcup_{z \in(0,1)}\left(\mathrm{B}\left(z, \delta_{z}\right) \bigcap U\right)$, $\mathrm{W}=\bigcup_{z \in(0,1)}\left(\mathrm{B}\left(z, \delta_{z}\right) \bigcap \mathrm{L}\right)$ (where $\mathrm{L}$ denotes the lower half-plane). Thus $N=$ $\mathrm{V} \cup(0,1) \bigcup \mathrm{W}$. Let $\mathrm{V}_{n}$ denote the component of $\mu_{n}\left(\mathrm{D}_{n}\right) \backslash(0,1)$ containing $\mathrm{V}$, $\mathrm{W}_{n}$ the component of $\mu_{n}\left(\mathrm{D}_{n}\right) \backslash(0,1)$ containing $\mathrm{W}$. Also, let $\mathrm{A}_{n}$ and $\mathrm{B}_{n}$ denote the components of $\partial \mu_{n}\left(\mathrm{D}_{n}\right) \backslash\left\{z_{n}^{\prime}, \infty\right\}$ containing 0 and 1 respectively.

Two observations will be useful.

Observation $1 \quad$ If $u_{n} \in \mathrm{A}_{n}, v_{n} \in \mathrm{B}_{n}$, with $\left|u_{n}\right| \leq k\left|z_{n}^{\prime}\right|,\left|v_{n}\right| \leq$ $k\left|z_{n}^{\prime}\right|$ for some $0<k<1$, then $\left|u_{n}-v_{n}\right| \geq \frac{1-k}{1+\epsilon_{n}}$.

Indeed, since $z_{n}^{\prime}$ is in the bounded component of $\partial \mu_{n}\left(\mathrm{D}_{n}\right) \backslash\{0,1\}$, from the definition of $d_{n}$ it follows that

$$
\left|u_{n}-v_{n}\right| \geq d_{n}\left|u_{n}-z_{n}^{\prime}\right| \geq d_{n}(1-k)\left|z_{n}^{\prime}\right| \geq d_{n}(1-k) \frac{1}{d_{n}\left(1+\epsilon_{n}\right)}=\frac{1-k}{1+\epsilon_{n}} .
$$

Since all domains concerned omit 0,1 and $\infty$, we may pass to a subsequence and assume that $\mathrm{V}_{n} \longrightarrow \mathrm{V}^{\prime}, \mathrm{W}_{n} \longrightarrow \mathrm{W}^{\prime}$ and $\mu_{n}\left(\mathrm{D}_{n}\right) \longrightarrow \mathrm{D}^{\prime}$ (with respect to any point in $\mathrm{V}, \mathrm{W}, N$ respectively), where $\mathrm{V}^{\prime}, \mathrm{W}^{\prime}$ and $\mathrm{D}^{\prime}$ are domains containing $\mathrm{V}, \mathrm{W}$ and $N$ respectively. It is easy to verify that $\mathrm{D}^{\prime}=\mathrm{V}^{\prime} \cup(0,1) \cup \mathrm{W}^{\prime}$. Moreover, since $\mathrm{V}_{n} \bigcap \mathrm{W}_{n}=\emptyset$ for all $n$, it easily follows that $\mathrm{V}^{\prime} \cap \mathrm{W}^{\prime}=\emptyset$.

Observation 2

$\mathrm{V}^{\prime}$ is non-Jordan or unbounded, and $\mathrm{W}^{\prime}$ is nonJordan or unbounded.

We verify the assertion for $\mathrm{V}^{\prime}$. Suppose, to the contrary that $\mathrm{V}^{\prime}$ is a bounded Jordan domain. Consider $\partial \mathrm{V}^{\prime} \backslash(0,1)$. For each $v \in \partial \mathrm{V}^{\prime} \backslash(0,1)$ there exists a sequence $\left\{v_{n}\right\}, v_{n} \in \partial \mathrm{V}_{n} \backslash(0,1)$, such that $v=\lim _{n \rightarrow \infty} v_{n}$. Let

$$
\begin{aligned}
& \mathrm{A}=\left\{v \in \partial \mathrm{V}^{\prime} \backslash(0,1): v=\lim _{n \rightarrow \infty} v_{n} \text { with } v_{n} \in \mathrm{A}_{n} \text { for infinitely many } n\right\}, \\
& \mathrm{B}=\left\{v \in \partial \mathrm{V}^{\prime} \backslash(0,1): v=\lim _{n \rightarrow \infty} v_{n} \text { with } v_{n} \in \mathrm{B}_{n} \text { for large enough } n\right\} .
\end{aligned}
$$

Then $0 \in \mathrm{A}, 1 \in \mathrm{B}$, so $\mathrm{A}$ and $\mathrm{B}$ are nonempty sets with $\mathrm{A} \cup \mathrm{B}=\partial \mathrm{V}^{\prime} \backslash(0,1)$ (since $\left.z_{n}^{\prime} \longrightarrow \infty\right)$. Since $\mathrm{V}^{\prime}$ is Jordan and $(0,1) \subseteq \partial \mathrm{V}^{\prime}$ it follows that $\partial \mathrm{V}^{\prime} \backslash(0,1)$ is connected. Thus, there exist $u \in \mathrm{A}$ and $v \in \mathrm{B}$ with $|u-v|<1 / 3$. From this and the definition of $\mathrm{A}$ and $\mathrm{B}$ we conclude that there exists an increasing sequence of natural numbers $\left\{n_{k}\right\}$ and $u_{n_{k}} \in \mathrm{A}_{n_{k}}, v_{n_{k}} \in \mathrm{B}_{n_{k}}$ such that $\lim _{k \rightarrow \infty} u_{n_{k}}=u$, 
$\lim _{k \rightarrow \infty} v_{n_{k}}=v$ and $\left|u_{n_{k}}-v_{n_{k}}\right|<1 / 3$ for each $k$. Since $\mathrm{V}^{\prime}$ is bounded and $\lim _{n \rightarrow \infty} z_{n}^{\prime}=\infty$ it follows that $\left|u_{n_{k}}\right| \leq \frac{1}{2}\left|z_{n_{k}}^{\prime}\right|$ and $\left|v_{n_{k}}\right| \leq \frac{1}{2}\left|z_{n_{k}}^{\prime}\right|$ for large enough $k$. Thus, by Observation $1,\left|u_{n_{k}}-v_{n_{k}}\right| \geq \frac{1}{2\left(1+\epsilon_{n_{k}}\right)}$ for large enough $k$. We have arrived at a contradiction. Hence, the observation is true.

We know that $(0,1) \subseteq \mathrm{D}^{\prime}, 0,1 \in \partial \mathrm{D}^{\prime}$. It is not hard to see that the components of $\mathrm{D}^{\prime} \backslash(0,1)$ are $\mathrm{V}^{\prime}$ and $\mathrm{W}^{\prime}$. From Observation 2 it follows that $\mathrm{D}^{\prime}$ is non-Jordan, proving the lemma.

We need the following definition. Suppose D is a simply connected domain. We say that a conformal mapping $g: \mathrm{D} \longrightarrow \overline{\mathbb{C}}$ is an extremal mapping for $\mathrm{D}$ if

(i) $\left\|\mathrm{S}_{g}\right\|_{\mathrm{D}}=\sigma(\mathrm{D})$;

(ii) $g$ cannot be extended to a quasiconformal mapping of $\overline{\mathbb{C}}$.

When $\mathrm{D}=U, g(z)=\log z$ is an extremal mapping for $\mathrm{D}$. Extremal mappings can also be easily demonstrated for angular sectors. It is not known whether every domain has an extremal mapping.

Lemma 4.3. Suppose D is a regulated quasidisk. Then there exists an extremal mapping for $\mathrm{D}$ or $\sigma(\mathrm{D})=\sigma\left(\mathrm{A}_{\alpha}\right)$, where $\alpha \pi \in(0,2 \pi)$ is such that $\partial \mathrm{D}$ has a corner of opening $\alpha \pi$.

Proof. Without loss of generality we may assume that D is bounded. Suppose that no extremal mapping for $\mathrm{D}$ exists. We can find a sequence of conformal mappings $g_{n}, g_{n}: \mathrm{D} \longrightarrow \overline{\mathbb{C}}$ such that:

(i) $\left\|\mathrm{S}_{g_{n}}\right\|_{\mathrm{D}} \longrightarrow \sigma(\mathrm{D})$; $\quad$ (ii) For each $n, \mathrm{D}_{n}=g_{n}(\mathrm{D})$ is a quasidisk and $d\left(\mathrm{D}_{n}\right) \longrightarrow 0$.

By Lemma 4.2 we may assume the existence of a sequence $\left\{\mu_{n}\right\}$ of Möbius transformations such that $0,1, \infty \in \partial \mu_{n}\left(\mathrm{D}_{n}\right)$ and $\mathrm{B}(1 / 2, \epsilon) \subseteq \mu_{n}\left(\mathrm{D}_{n}\right)$ for each $n$, for some $\epsilon>0$; and $\mu_{n}\left(\mathrm{D}_{n}\right) \longrightarrow \mathrm{D}^{\prime}$ with respect to $1 / 2$ where $\mathrm{D}^{\prime}$ is some non-Jordan domain.

Let $r_{n}$ denote the distance of $g_{n}^{-1} \circ \mu_{n}^{-1}(1 / 2)$ from $\partial \mathrm{D}$ and let $z_{n} \in \partial \mathrm{D}$ be a point for which $\left|z_{n}-g_{n}^{-1} \circ \mu_{n}^{-1}(1 / 2)\right|=r_{n}$. Choose a similarity transformation $\lambda_{n}$ that maps $\mathrm{B}\left(g_{n}^{-1} \circ \mu_{n}^{-1}(1 / 2), r_{n}\right)$ onto $\mathrm{B}$ with $\lambda_{n}\left(g_{n}^{-1} \circ \mu_{n}^{-1}(1 / 2)\right)=0$ and $\lambda_{n}\left(z_{n}\right)=1$. Since the domains $\lambda_{n}(\mathrm{D})$ all contain $\mathrm{B}$ and omit 1 and $\infty$ we may assume, by extracting a subsequence, that $\lambda_{n}(\mathrm{D}) \longrightarrow \mathrm{D}_{0}$ with respect to 0 , where $\mathrm{D}_{0}$ is some domain containing $\mathrm{B}$.

By Lemma 4.1, $\mathrm{D}_{0}$ is Möbius equivalent to $\mathrm{D}$ or to $U$ or to $\mathrm{A}_{\alpha}$, an angular sector associated with some corner of $\partial \mathrm{D}$. In any case, $\sigma\left(\mathrm{D}_{0}\right) \geq \sigma(\mathrm{D})$ (see Lemma 3.3). Now let $f_{n}=\mu_{n} \circ g_{n} \circ \lambda_{n}^{-1}$ in $\lambda_{n}(\mathrm{D})$. (For any $z \in \overline{\mathrm{D}}_{0}, f_{n}(z)$ is defined for large enough $n$.) Since $0,1, \infty \notin f_{n}\left(\lambda_{n}(\mathrm{D})\right)=\mu_{n}\left(\mathrm{D}_{n}\right)$ by assumption, the family $\left\{f_{n}\right\}$ is normal in $\mathrm{D}_{0}$. We may therefore suppose that $f_{n} \longrightarrow f$ locally uniformly on $\mathrm{D}_{0}$, where $f: \mathrm{D}_{0} \longrightarrow \overline{\mathbb{C}}$. Since

$$
f_{n}(0)=\mu_{n} \circ g_{n} \circ \lambda_{n}^{-1}(0)=\mu_{n} \circ g_{n}\left(g_{n}^{-1} \circ \mu_{n}^{-1}\left(\frac{1}{2}\right)\right)=\frac{1}{2},
$$


$\mathrm{B}(1 / 2, \epsilon) \subseteq f_{n}\left(\lambda_{n}(\mathrm{D})\right)=\mu_{n}\left(\mathrm{D}_{n}\right)$ for all $n$ and $\mu_{n}\left(\mathrm{D}_{n}\right) \longrightarrow \mathrm{D}^{\prime}$ with respect to $1 / 2$, we conclude that $f$ maps $\mathrm{D}_{0}$ conformally onto $\mathrm{D}^{\prime}$. Since $\mathrm{D}^{\prime}$ is non-Jordan, $\left\|\mathrm{S}_{f}\right\|_{\mathrm{D}_{0}} \geq \sigma\left(\mathrm{D}_{0}\right) \geq \sigma(\mathrm{D})$. On the other hand, we have

$$
\begin{aligned}
\left\|\mathrm{S}_{f}\right\|_{\mathrm{D}_{0}} \leq \liminf _{n \rightarrow \infty}\left\|\mathrm{S}_{f_{n}}\right\|_{\lambda_{n}(\mathrm{D})} & \\
& =\liminf _{n \rightarrow \infty}\left\|\mathrm{S}_{\mu_{n} \circ g_{n} \circ \lambda_{n}^{-1}}\right\|_{\lambda_{n}(\mathrm{D})}=\liminf _{n \rightarrow \infty}\left\|\mathrm{S}_{g_{n}}\right\|_{\mathrm{D}}=\sigma(\mathrm{D}) .
\end{aligned}
$$

Thus, $\left\|\mathrm{S}_{f}\right\|_{\mathrm{D}_{0}}=\sigma(\mathrm{D})=\sigma\left(\mathrm{D}_{0}\right)$. Since $f\left(\mathrm{D}_{0}\right)=\mathrm{D}^{\prime}$ and $\mathrm{D}^{\prime}$ is non-Jordan, we conclude that there exists an extremal mapping for $\mathrm{D}_{0}$. Thus, there exists an extremal mapping for $\mathrm{D}$ (if $\mathrm{D}_{0}$ is Möbius equivalent to $\mathrm{D}$ or if $\mathrm{D}_{0}$ and consequently $\mathrm{D}$ is a half-plane) or $\sigma(\mathrm{D})=\sigma\left(\mathrm{A}_{\alpha}\right)$ for some angular sector $\mathrm{A}_{\alpha}$ associated with a corner of $\partial \mathrm{D}$ (if $\mathrm{D}_{0}$ is Möbius equivalent to $\mathrm{A}_{\alpha}$ ). The lemma is proved.

If $\sigma(\mathrm{D})=\sigma\left(\mathrm{A}_{\alpha}\right)$ for some corner of opening $\alpha \pi$ of $\partial \mathrm{D}$ with $0<\alpha \leq 1$, then $\alpha \pi$ is the smallest corner of $\partial \mathrm{D}$. Now we turn to the necessary condition portion of the main result.

Theorem 4.4. Suppose that D is a regulated domain with convex corners and that $h$ maps B conformally onto D. If D is a Nehari disk, then $\lim \sup \left|\mathrm{S}_{h}(z)\right|\left(1-|z|^{2}\right)^{2}=\left\|\mathrm{S}_{h}\right\|_{\mathrm{B}}$. $|z| \rightarrow 1$

Proof. Suppose D is a Nehari disk i.e., $\sigma(\mathrm{D})=2-\left\|\mathrm{S}_{h}\right\|_{\mathrm{B}}$. According to Lemma 4.3, there are two cases. Consider the first case i.e., assume that there exists a conformal mapping $g: \mathrm{D} \longrightarrow \overline{\mathbb{C}}$ with $\left\|\mathrm{S}_{g}\right\|_{\mathrm{D}}=\sigma(\mathrm{D})$ which has no quasiconformal extension to $\overline{\mathbb{C}}$. Since $\mathrm{D}$ is a Nehari disk, from Theorem 1.2 it follows that $g(\mathrm{D})$ is either a Jordan domain but not a quasidisk or is Möbius equivalent to a parallel strip. Accordingly, $g \circ h(\mathrm{~B})=g(\mathrm{D})$ fits one of these two descriptions. From Theorem 1.3 we conclude that $\limsup \left|\mathrm{S}_{g \circ h}(z)\right|\left(1-|z|^{2}\right)^{2} \geq 2$, so

$$
|z| \rightarrow 1
$$

$$
\begin{aligned}
2 \leq \limsup _{w \rightarrow \partial \mathrm{D}}\left|\mathrm{S}_{g}(w)\right| \rho_{\mathrm{D}}^{-2}(w)+\limsup _{|z| \rightarrow 1}\left|\mathrm{~S}_{h}(z)\right|\left(1-|z|^{2}\right)^{2} & \\
& \leq\left\|\mathrm{S}_{g}\right\|_{\mathrm{D}}+\limsup _{|z| \rightarrow 1}\left|\mathrm{~S}_{h}(z)\right|\left(1-|z|^{2}\right)^{2} .
\end{aligned}
$$

Therefore, $\left\|\mathrm{S}_{h}\right\|_{\mathrm{B}}=2-\sigma(\mathrm{D})=2-\left\|\mathrm{S}_{g}\right\|_{\mathrm{D}} \leq \limsup _{|z| \rightarrow 1}\left|\mathrm{~S}_{h}(z)\right|\left(1-|z|^{2}\right)^{2}$ so we conclude that $\limsup _{|z| \rightarrow 1}\left|\mathrm{~S}_{h}(z)\right|\left(1-|z|^{2}\right)^{2}=\left\|\mathrm{S}_{h}\right\|_{\mathrm{B}}$, which proves the theorem in this case.

Now suppose that $\sigma(\mathrm{D})=\sigma\left(\mathrm{A}_{\alpha}\right)$ for some corner of opening $\alpha \pi$ of $\partial \mathrm{D}$. Since $\mathrm{D}$ has convex corners only, from Lemma 3.3 we have $\sigma(\mathrm{D})=\min _{k} 2 \alpha_{k}^{2}=$ $2-\max _{k}\left(2-2 \alpha_{k}^{2}\right)$ (where $\alpha_{k} \pi$ are the corners of $\left.\partial \mathrm{D}\right)$. Because $\mathrm{D}$ is a Nehari disk, $\left\|\mathrm{S}_{h}\right\|_{\mathrm{B}}=\max _{k}\left(2-2 \alpha_{k}^{2}\right)$. Now, since $\mathrm{D}$ is regulated from Lemma 3.2 we 
conclude that $\limsup _{|z| \rightarrow 1}\left|\mathrm{~S}_{h}(z)\right|\left(1-|z|^{2}\right)^{2}=\left\|\mathrm{S}_{h}\right\|_{\mathrm{B}}$. This completes the proof of the theorem.

We remark that, as a consequence of Theorem 4.4, domains with smooth boundaries, other than disks in $\overline{\mathbb{C}}$, cannot be Nehari disks.

\section{Acknowledgement}

The author wishes to use this opportunity to thank the referee for valuable suggestions used to improve the presentation in this paper.

\section{References}

[1] L. V. Ahlfors, Quasiconformal reflections, Acta Math. 109 (1963), 291-301.

[2] L. V. Ahlfors, Complex Analysis, McGraw-Hill, New York, second edition, 1966.

[3] L. V. Ahlfors, G. Weill, A uniqueness theorem for Beltrami equations, Proc. Amer. Math Soc. 13 (1962), 975-978.

[4] D. Calvis, The inner radius of univalence of normal circular triangles and regular polygons, Complex Variables Theory Appl. 4 (1985), 295-304.

[5] F. W. Gehring, Univalent functions and the Schwarzian derivative, Comment. Math. Helv. 52 (1977), 561-572.

[6] F. W. Gehring, Characteristic Properties of Quasidisks, Les Presses de l'Université de Montréal, Montreal, 1982.

[7] F. W. Gehring, Ch. Pommerenke, On the Nehari univalence criterion and quasicircles, Comment. Math. Helv. 59 (1984), 226-242.

[8] E. Hille, Remarks on a paper by Zeev Nehari, Bull. Amer. Math. Soc. 55 (1949), 552-553.

[9] M. Lehtinen, On the inner radius of univalency for non-circular domains, Ann. Acad. Sci. Fenn. Ser. A 1(5) (1980), 45-47.

[10] O. Lehto, Remarks on Nehari's theorem about the Schwarzian derivative and schlicht functions, Journal d'Analyse Math. 36 (1979), 184-190.

[11] O. Lehto, Univalent Function and Teichmüller Spaces, Springer-Verlag, New York, 1987.

[12] L. Miller-Van Wieren, Univalence criteria for classes of rectangles and equiangular hexagons, Ann. Acad. Sci. Fenn. Math. 22 (1997), 407-424.

[13] L. Miller-Van Wieren, Univalence Criteria for Analytic Functions, PhD thesis, University of Michigan, 1994.

[14] Z. Nehari, The Schwarzian derivative and Schlicht functions, Bull. Amer. Math. Soc. 55 (1949), 545-551.

[15] V. Paatero, Über die konforme Abbildung von Gebieten deren Ränder von beschränkter Drehung sind, Ann. Acad. Sci. Fenn. Ser. A 33(9) (1931), 1-11.

[16] Ch. Pommerenke, Boundary Behaviour of Conformal Maps, Grundlehren der mathematischen Wissenschaften, A Series of Comprehensive Studies in Mathematics. Springer-Verlag, New York, 1992.

[17] W. Rudin, Principles of Mathematical Analysis, McGraw-Hill, New York, third edition, 1976. 
Leila Miller-Van Wieren

Department of Mathematics

University of Texas at Austin

Austin, TX 78712-1082

USA

e-mail: leila@math.utexas.edu

(Received: March 7, 1996; revised version: July 15, 1999) 